\title{
Highly Dispersed Cu-Base Catalyst Derived from Layered Double Hydroxides for CO Hydrogenation
}

\author{
Xinyou Han1,2, Kegong Fang1, Minggui Lin', Yuhan Sun ${ }^{1,3}$ \\ ${ }^{1}$ State Key Laboratory of Coal Conversion, Institute of Coal Chemistry, Chinese Academy of Sciences, Taiyuan, \\ China \\ ${ }^{2}$ University of the Chinese Academy of Sciences, Beijing, China \\ ${ }^{3}$ Shanghai Advanced Research Institute, Chinese Academy of Sciences, Shanghai, China \\ Email: xy han@live.com
}

Received February 2014

\begin{abstract}
Highly dispersed Cu-base catalyst has been prepared via thermal decomposition of layered double hydroxides precursors. The XRD pattern and the HRTEM images of the as prepared catalyst confirmed the high dispersion of $\mathrm{Cu}$ and $\mathrm{Fe}$ ions. Results show that the catalyst has a relatively high selectivity of alkanes at low temperature.
\end{abstract}

Keywords

Highly Dispersed, LDHs, CO Hydrogenation, Cu Base

\section{Introduction}

Cu-based catalysts containing active metal toward Fischer-Tropsch synthesis such as $\mathrm{Cu}-\mathrm{Fe}$ based catalyst, is considered as one of the most promising catalysts for higher alcohols synthesis from syngas [1-3]. Most researchers considered the synergistic effect between $\mathrm{Cu}$ and Fe played a key role in the higher alcohol synthesis (HAS) [4]. However, the copper sintering at higher temperature has a negative influence on the synergistic effect. In the layer structure of the layered double hydroxides (LDHs), metal ions mixed with each other homogeneously [5], which might facilitate the synergetic effect of the active metal particles and inhibit the sintering of the metals. Thus, in this paper catalyst derived from layered double hydroxides containing highly dispersed $\mathrm{Cu}$ has been prepared by hydrothermal method and its application in the CO hydration has been examined.

\section{Experiments}

Highly dispersed Cu-base catalyst was prepared according to the reference [5] with some modification. In a typical process, $1.21 \mathrm{~g} \mathrm{Cu}\left(\mathrm{NO}_{3}\right)_{2} \cdot 3 \mathrm{H}_{2} \mathrm{O}, 2.02 \mathrm{~g} \mathrm{Fe}\left(\mathrm{NO}_{3}\right)_{3} \cdot 9 \mathrm{H}_{2} \mathrm{O}, 5 \mathrm{~g} \mathrm{Al}\left(\mathrm{NO}_{3}\right)_{3} \cdot 9 \mathrm{H}_{2} \mathrm{O}$ and $12.8 \mathrm{~g} \mathrm{Mg}\left(\mathrm{NO}_{3}\right)_{2} \cdot 6 \mathrm{H}_{2} \mathrm{O}$ were dissolved together in $400 \mathrm{~mL}$ deionized water (molar ratio, MII/MIII = 3), which was referred as solution A. Solution B was the mixture of $\mathrm{K}_{2} \mathrm{CO}_{3}$ and $\mathrm{KOH}$ with concentration of 0.25 and $0.8 \mathrm{~mol} / \mathrm{L}$. Solutions A and B

How to cite this paper: Han, X.Y., Fang, K., Lin, M.G. and Sun, Y.H. (2014) Highly Dispersed Cu-Base Catalyst Derived from Layered Double Hydroxides for CO Hydrogenation. Journal of Materials Science and Chemical Engineering, 2, 1-3.

http://dx.doi.org/10.4236/msce.2014.26001 
were simultaneously added into a glass reactor under vigorous stirring at room temperature and a $\mathrm{pH}$ value of 9.5.

The slurry was aged at $393 \mathrm{~K}$ for $20 \mathrm{~h}$, filtered off, and washed thoroughly with distilled water. The precipitate was then dried at $353 \mathrm{~K}$ for $12 \mathrm{~h}$ and identified as CuFe-LDH. The layered structure of prepared CuFe-LDH sample was confirmed by XRD (see Figure 1).

These Cu-containing layered double hydroxides were calcined at $573 \mathrm{~K}$ in a stationary air for $4 \mathrm{~h}$ and the product catalysts were identified as $\mathrm{CuFeMgAl}$. The catalytic performance was tested in a fixed-bed reactor.

\section{Results and Discussions}

The XRD patterns of the precursor before and after calcination are shown in Figure 1. The XRD patterns before calcination illustrate the typical peaks for LDHs. Peaks at 11.6, 23.3, 34.7, 39.3, 46.7, 60.5, and 61.8 are observed in the precursor, which are assigned to the (003), (006), (012), (015), (018), (110), and (113) diffractions of LDHs.

Further more, from the XRD pattern no clear peaks of $\mathrm{Cu}$, $\mathrm{Fe}$ or $\mathrm{CuFe}$ oxide species are found which mean that the $\mathrm{Cu}$ and $\mathrm{Fe}$ ions are highly dispersed in the precursor. After calcination, peak at 13 was assigned as the remain layered structure and peaks at 60.5 and 61.8 are the remain structure of LDHs within the layers which we can also see at Figure 2(b).

Figure 2(a) shows the cross-section of layered platelet in CuFeMgAl, from which it can be found that the layered structure of hydrotalcite. Agreed with the result of XRD pattern, no $\mathrm{Cu}$ or Fe oxide particles can be found in the plates which indicate the highly dispersion of metal ions in the LDHs.

The catalytic performance of the catalyst towards $\mathrm{CO}$ hydrogenation was examined under the following reaction conditions: 4.0 MPa, GHSV of $5000 \mathrm{~h}^{-1}, \mathrm{n}\left(\mathrm{H}_{2}\right) / \mathrm{n}(\mathrm{CO})=2.0$ and the results are shown in Table 1. It could be seen that the main products at low temperature was alkanes with selectivity of $93.8 \mathrm{wt} . \%$. Along with the rise of temperature, the selectivity of alkanes fall down and the selectivity of alcohols changed from 1.09 wt.\% at

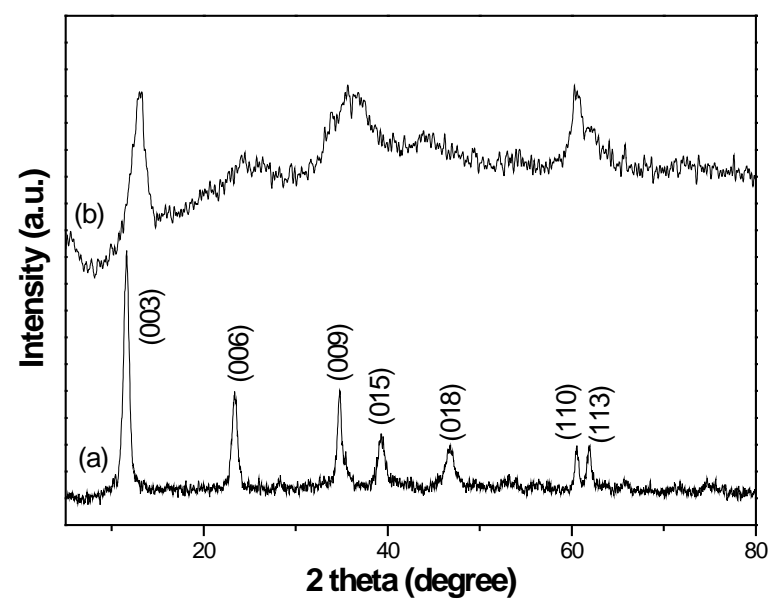

Figure 1. XRD patterns of the precursor before (a) and after (b) calcinations.

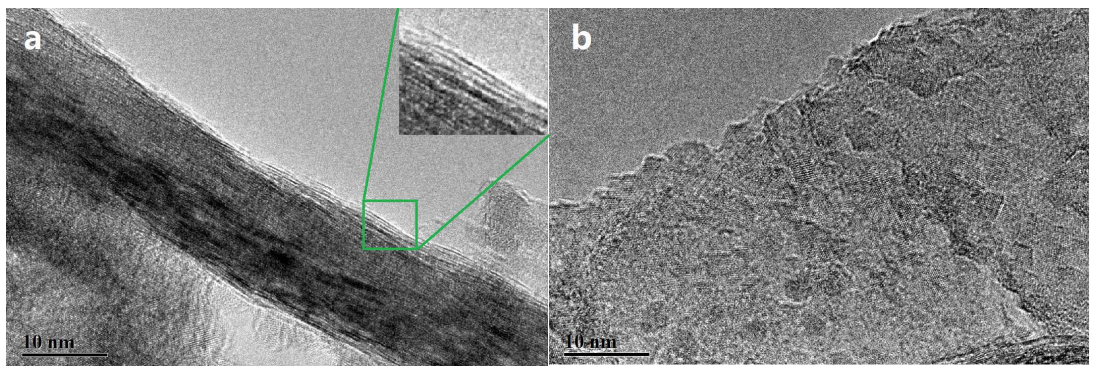

Figure 2. Layered structure of the calcinlated sample, stand up (a) and lie down (b). 
Table 1. Catalytic results of layered double hydroxides (LDHs) supported catalyst.

\begin{tabular}{|c|c|c|c|c|c|c|c|c|}
\hline \multirow{2}{*}{ Catalyst } & \multirow{2}{*}{$\mathbf{T}(\mathbf{K})$} & \multirow{2}{*}{ CO conversion (\%) } & \multirow{2}{*}{$\frac{\text { STY }_{\text {ROH }}}{\text { (g/g/h) }}$} & \multicolumn{3}{|c|}{ Selectivity (wt.\%) } & \multicolumn{2}{|c|}{ Alcohol distribution (wt.\%) } \\
\hline & & & & ROH & CHn & $\mathrm{CO}_{2}$ & $\mathrm{C1OH}$ & $\mathrm{C} 2+\mathrm{OH}$ \\
\hline \multirow{4}{*}{ CuFeMgAl } & 493 & 8.05 & 0.00 & 1.09 & 93.80 & 5.11 & 59.61 & 40.39 \\
\hline & 513 & 19.39 & 0.01 & 3.28 & 83.86 & 12.86 & 61.47 & 38.53 \\
\hline & 533 & 32.28 & 0.06 & 9.91 & 67.79 & 22.30 & 58.86 & 41.14 \\
\hline & 553 & 54.99 & 0.11 & 9.04 & 54.03 & 36.93 & 53.63 & 46.37 \\
\hline
\end{tabular}

Reaction conditions: $\mathrm{H}_{2} / \mathrm{CO}=2.0$, GHSV $=5000 \mathrm{~h}^{-1}, \mathrm{P}=4.0 \mathrm{MPa}$.

$493 \mathrm{~K}$ to $9.91 \mathrm{wt} . \%$ at $533 \mathrm{~K}$. And at $553 \mathrm{~K}$ the selectivity of alcohols slightly fell may be due to the sintering of $\mathrm{Cu}$.

\section{Conclusion}

In summary, this work confirmed that bimetallic highly dispersed Cu-base catalysts could be prepared via thermal decomposition of layered double hydroxides precursors. This is attributed to the layered structure of hydrotalcite which could efficiently inhibit the sintering of the $\mathrm{Cu}$ and Fe ions. Furthermore, at low temperature the main products of the catalyst were alkanes and as temperature grows the selectivity of alcohols becomes higher.

\section{References}

[1] Lin, M., Fang, K., Li, D. and Sun, Y. (2008) CO Hydrogenation to Mixed Alcohols over Co-Precipitated Cu-Fe Catalysts. Catalysis Communications, 9, 1869-1873. http://dx.doi.org/10.1016/j.catcom.2008.03.004

[2] Lin, M., Fang, K., Li, D. and Sun, Y. (2008) Phase Transformation in Cu-Fe Based Catalyst during CO Hydrogenation. Chinese Journal of Catalysis, 29, 559-565.

[3] Fang, K., Li, D., Lin, M., Xiang, M., Wei, W. and Sun, Y. (2009) A Short Review of Heterogeneous Catalytic Process for Mixed Alcohols Synthesis via Syngas. Catalysis Today, 147, 133-138.

[4] Gao, W., Zhao, Y.F., Liu, J.M., Huang, Q.W., He, S., Li, C.M., Zhao, J.W. and Wei, M. (2013) Catalytic Conversion of Syngas to Mixed Alcohols over CuFe-Based Catalysts Derived from Layered Double Hydroxides. Catalysis Science \& Technology, 5, 1324-1332.

[5] Yuan, Z., Wang, L., Wang, J., Xia, S., Chen, P., Hou, Z. and Zheng, X. (2011) Hydrogenolysis of Glycerol over Homogenously Dispersed Copper on Solid Base Catalysts. Applied Catalysis B: Environmental, 101, 431-440. http://dx.doi.org/10.1016/j.apcatb.2010.10.013 